\title{
Quality of Care for HIV/AIDS and for Primary Prevention by HIV Specialists and Nonspecialists
}

\author{
Raphael J. Landovitz, MD, MSc, Katherine A. Desmond, MS, \\ Jennifer L. Gildner, MS, and Arleen A. Leibowitz, PhD $^{2}$
}

\begin{abstract}
The role of HIV specialists in providing primary care to persons living with HIV/AIDS is evolving, given their increased incidence of comorbidities. Multivariate logit analysis compared compliance with sentinel preventive screening tests and interventions among publicly insured Californians with and without access to HIV specialists in 2010. Quality-of-care indicators [visit frequency, CD4 and viral load (VL) assessments, influenza vaccine, tuberculosis (TB) testing, lipid profile, glucose blood test, and Pap smears for women] were related to patient characteristics and provider HIV caseload. There were 9377 adult Medicare enrollees $(71 \%$ also had Medicaid coverage) and 2076 enrollees with only Medicaid coverage. Adjusted for patient characteristics, patients seeing providers with greater HIV caseloads ( $>50 \mathrm{HIV}$ patients) were more likely to meet visit frequency guidelines in both Medicare [98\%; confidence interval (CI 97.5-98.2) and Medicaid (97\%; CI 96.298.0), compared to 60\% (CI 57.1-62.3) and 45\% (CI 38.3-50.4), respectively, seeing providers without large HIV caseloads $(p<0.001)$. Patients seeing providers with larger caseloads were significantly more likely to have CD4 $(p<0.001)$, VL $(p<0.001)$, and TB testing $(p<0.05)$. A larger percentage of patients seeing largevolume Medicare providers received influenza vaccinations. Provider caseload was unrelated to lipid or glucose assessments or Pap Smears for women. Patients with access to large-volume providers were more likely to meet clinical guidelines for visits, CD4, VL, tuberculosis testing, and influenza vaccinations, and were not less likely to receive primary preventive care. Substantial insufficiencies remain in both monitoring to assess viral suppression and in preventive care.
\end{abstract}

\section{Introduction}

$\mathbf{T}$ HE LARGE AND IMPORTANT reductions in morbidity and mortality for people living with HIV/AIDS (PLWHA), which have come about due to the widespread adoption of antiretroviral therapy (ART), have increased life expectancy for PLWHA. ${ }^{1,2}$ This increased longevity obligates increased attention to health problems associated with aging. When an expert panel of the HIV Medicine Association of the Infectious Diseases Society of America updated its recommended guidelines for HIV care in 2009, it noted that the improved survival of PLWHA since its 2004 recommendations enhanced the importance of screening for these nonHIV chronic diseases. ${ }^{3}$

As survival for PLWHA increases and HIV treatment continues to minimize pill burden and toxicity, medical care for PLWHA not only needs to optimize HIV-specific treatment but also to include more primary prevention activities, particularly for the common comorbidities of aging, which PLWHA may experience at earlier ages than the general population due to immune exhaustion and chronic inflammation engendered by even well-controlled/treated HIV infection. ${ }^{4}$ Reflecting this need, in 2014, the Health Resources and Services Administration (HRSA) expanded its list of HIV/AIDS Care Performance Measures to include viral load (VL) monitoring, influenza immunization, and hepatitis B vaccination, in addition to the core HIV clinical performance measures (HIV VL suppression; prescription of ART, appropriate number and spacing of medical evaluations; Pneumocystis jiroveci pneumonia prophylaxis). ${ }^{5}$ The expanded list of performance measures also encompasses enhanced screening for conditions affecting the general population

\footnotetext{
${ }^{1}$ Division of Infectious Diseases, UCLA David Geffen School of Medicine, UCLA Center for Clinical AIDS Research and Education, Los Angeles, California.

${ }^{2}$ Department of Public Policy, UCLA Luskin School of Public Affairs, Los Angeles, California.
} 
(e.g., annual influenza vaccination and annual fasting lipid panel for all PLWHA who are prescribed ART, not just men older than 35 years), as well as screening for health conditions that are more prevalent among PLWHA, including cervical cancer, chlamydia, gonorrhea, syphilis, hepatitis B and $\mathrm{C}$, and tuberculosis (TB). ${ }^{6,7}$ (HRSA 2014 contains a complete list of performance measures.)

Nonetheless, the rates at which PLWHA are screened for many of these conditions are lower than screening rates in the general population, although the prevalence of these conditions among PLWHA is greater. ${ }^{8,9}$ For example, only $58 \%$ of HIV-positive women in Ottawa were screened for cervical CA within a 3 -year period ${ }^{10,11}$ and only $55.6 \%$ of patients with HIV had had at least one colorectal cancer screening. 9

It is well established that providers who treat greater numbers of HIV/AIDS patients deliver higher quality HIV treatment. HIV/AIDS patient survival, ${ }^{12-15}$ appropriate initiation and monitoring of ART treatment, ${ }^{13,16,17}$ and prescribing a medically recommended regimen of ART if any ART is prescribed, all depend on the provider's experience in treating HIV. ${ }^{18} \mathrm{HIV}$-specific patient volume/experience has a greater effect on HIV quality measures and HIVspecific knowledge than provider type (physician vs. midlevel provider) or specialty (infectious disease specialist vs. generalist). ${ }^{19-21}$

There is conflicting evidence on whether HIV specialists are as effective as generalists in providing increasingly important primary prevention to their HIV/AIDS patients. A bill recently introduced in the California legislature (AB2372) aims to better integrate HIV treatment with primary care by requiring state-regulated health insurance plans to include HIV specialists as primary care providers for PLWHA. Although many patients have a preference for "one stop shopping" where the same provider serves as both HIV specialist and primary physician, it has been unclear whether HIV specialty providers best serve the need for primary care services that an ever-aging HIV population requires. Some studies find that although HIV specialists deliver higher quality HIV-specific care, they are less likely to conduct screening for comorbidities, including cancer. ${ }^{10,22,23}$ However, there is also some evidence that the most experienced HIV providers are more likely to provide preventive care and testing for HIV-related conditions. ${ }^{24}$ For example, a 1995 study found that providers with the most HIV experience delivered more HIV-specific preventive screenings for coinfections and were more likely to have patients who received pneumococcal vaccination. ${ }^{25}$ Koethe et al. did not find statistically significant differences between general internal medicine and infectious disease physicians in screening rates for cervical cancer among HIV-positive women. ${ }^{26}$

To provide evidence on the delivery of preventive services to PLWHA, this article analyzes the impact of provider experience on the delivery of preventive services to PLWHA for a large population of PLWHA in California who were enrolled in Medicaid or Medicare in 2010.

\section{Methods}

Data

Medicare and Medicaid claims data for HIV-positive Californians were acquired through a confidential data use agreement with the Centers for Medicare and Medicaid Services (CMS). An algorithm that required an HIV diagnosis in two outpatient claims or one inpatient claim was used to identify adult beneficiaries with strong evidence of HIV. ${ }^{27}$ Our analyses included only fee-for-service Medicare and Medicaid enrollees because available data for Medicare managed care enrollees lack diagnosis fields needed to confirm HIV status. Medicaid-only beneficiaries (not dually enrolled in both Medicaid and Medicare) with visits to federally qualified health centers or rural health centers were excluded due to limited coding of procedures in these claims. To exclude persons newly diagnosed, for whom the full-year assessment of care would be inappropriate, we restricted our analyses to individuals enrolled full-year in both 2009 and 2010, with an HIV diagnosis code recorded in the claims data before 2009 (looking back to 2007-2008). We combine all Medicare beneficiaries, including "duals" into an "any Medicare" group, since Medicare is the primary payer for both and provides access to a wider range of providers than Medicaid. Any Medicare and Medicaid-only beneficiaries were analyzed separately.

\section{Outcome variables}

Eight different quality-of-care outcomes were assessed, based on recommended HIV care guidelines in 2009, which are relevant for our 2010 data. $^{3}$ Use of ART has been examined previously and is not included in this analysis. ${ }^{18}$ Guidelines recommend evaluation and management (E\&M) visits, and CD4 testing and VL testing occur at least twice a year, at least 3 months apart. Outpatient E\&M claims were defined by CPT-4 code, and type and place of service. To allow for some flexibility, we assessed if enrollees had at least two visits, spaced at least 80 days apart in 2010 , or one visit in 2010 and another in 2009 at least 80 days but not more than 365 days prior. Receipt of CD4 and VL tests was identified by procedure codes in the claims data.

Procedure and/or diagnosis codes in the 2010 claims data determined whether enrollees received each of four preventive services recommended annually for PLWHA: an influenza vaccine, TB testing (PPD or interferon gamma release assay), lipid profile, and glucose blood test. We assessed whether women received an annual cervical cancer screening.

\section{Individual characteristics}

Enrollment data provided information on age category $(<30,30-39,40-49,50-64$, and 65+), enrollee-reported race/ ethnicity (non-Hispanic white, African American, Hispanic/ Latino, and other), and gender. Diagnoses of adjustment or anxiety disorders, mood disorders, schizophrenia or other psychotic disorders, or other mental health disorders were identified using the Mental Health and Substance Abuse Clinical Classifications Software. ${ }^{28}$ Comorbidities were defined by examining ICD-9 diagnoses, and classified according to the Charlson Comorbidity Index, ${ }^{29}$ a commonly used method of classifying comorbidities. Using this index, the number of comorbidities was determined for each individual. The Medicare analyses included an indicator for enrollment in Medicare-only vs. dual Medicare-Medicaid enrollment. 


\section{Provider characteristics}

Each provider's caseload of publicly insured patients with HIV was assessed by counting unique HIV-positive Medicare and Medicaid enrollees seen for HIV-related care during 2010. Providers with fewer than five HIV patients were deemed non-HIV specialists. HIV-related care was defined as E\&M visits with an ICD-9 diagnosis code of symptomatic (042) or asymptomatic (V08) HIV in the first diagnosis field of the claim. To demonstrate a patient's known access to an experienced HIV provider, we assigned to each beneficiary the caseload category of the highest volume provider with whom the patient had any E\&M visit (not necessarily HIV related). Those with no E\&M visits in 2010 fell into the lowest volume group of access to a provider with $<5$ HIV patients.

\section{Statistical analysis}

We estimated logistic regressions for the Medicare and Medicaid samples to relate meeting E\&M guidelines to individual and provider characteristics. Logistic regression models for the seven other outcomes were fit on the sample that met the E\&M guideline. Wald chi-square $\left(\chi^{2}\right)$ tests on the logistic regression coefficients indicate significant differences between specific categories and a reference group, holding other factors constant. Recycled predictions were

Table 1. Sample Characteristics and Observed Percentages of California Medicare and Medicaid People Living with HIV/AIDS Meeting Visit Guidelines in 2010

\begin{tabular}{|c|c|c|c|c|c|c|}
\hline \multirow[b]{2}{*}{ Group } & \multicolumn{3}{|c|}{$\begin{array}{c}\text { Medicare enrollees } \\
\text { Guideline visits }\end{array}$} & \multicolumn{3}{|c|}{$\begin{array}{l}\text { Medicaid-only enrollees } \\
\text { Guideline visits }\end{array}$} \\
\hline & $\mathrm{n}(\%)$ in group & $\mathrm{n}(\%$ of group) & $\chi^{2} \mathrm{p}$ Value & $\mathrm{n}(\%)$ in group & $\mathrm{n}(\%$ of group) & $\chi^{2} \mathrm{p}$ Value \\
\hline Total & $9377(100.0)$ & 8705 (92.8) & & $2076(100.0)$ & $1867(89.9)$ & \\
\hline \multicolumn{7}{|l|}{ Enrollment } \\
\hline Medicare only & $2727(29.1)$ & $2600(95.3)$ & $<0.001$ & & & \\
\hline Dual & $6650(70.9)$ & $6105(91.8)$ & & & & \\
\hline \multicolumn{7}{|l|}{ Age } \\
\hline$<30$ & $71(0.8)$ & $59(83.1)$ & & $79(3.8)$ & $73(92.4)$ & \\
\hline $30-39$ & $524(5.6)$ & $468(89.3)$ & & $195(9.4)$ & $175(89.7)$ & \\
\hline $40-49$ & $3173(33.8)$ & 2905 (91.6) & $<0.001$ & $817(39.4)$ & $727(89.0)$ & 0.55 \\
\hline $50-65$ & $4246(45.3)$ & 3987 (93.9) & & $975(47.0)$ & $884(90.7)$ & \\
\hline $65+$ & $1363(14.5)$ & $1286(94.4)$ & & & & \\
\hline \multicolumn{7}{|l|}{ Gender } \\
\hline Male & 8427 (89.9) & $7837(93.0)$ & 0.07 & $1515(73.0)$ & $1349(89.0)$ & 0.03 \\
\hline Female & $950(10.1)$ & $868(91.4)$ & & $561(27.0)$ & $518(92.3)$ & \\
\hline \multicolumn{7}{|l|}{ Race } \\
\hline White & $5541(59.1)$ & $5181(93.5)$ & & $801(38.6)$ & 708 (88.4) & \\
\hline African American & $1639(17.5)$ & $1520(92.7)$ & 0.004 & $685(33.0)$ & $625(91.2)$ & 0.15 \\
\hline Hispanic & $1797(19.2)$ & $1635(91.0)$ & & 372 (17.9) & $332(89.2)$ & \\
\hline Other & $400(4.3)$ & $369(92.3)$ & & $218(10.5)$ & $202(92.7)$ & \\
\hline \multicolumn{7}{|c|}{ Most experienced provider has } \\
\hline $50+$ HIV patients & $5910(63.0)$ & $5776(97.7)$ & & $1372(66.1)$ & $1333(97.2)$ & \\
\hline 5-49 HIV patients & $2225(23.7)$ & $2173(97.7)$ & $<0.001$ & $438(21.1)$ & $418(95.4)$ & $<0.001$ \\
\hline$<5$ HIV patients & $1242(13.2)$ & $756(60.9)$ & & $266(12.8)$ & $116(43.6)$ & \\
\hline \multicolumn{7}{|l|}{ Comorbidities } \\
\hline None & $5647(60.2)$ & $5126(90.8)$ & & $1330(64.1)$ & $1175(88.3)$ & \\
\hline One & $2463(26.3)$ & $2339(95.0)$ & & $548(26.4)$ & $501(91.4)$ & \\
\hline Two & $794(8.5)$ & 777 (97.9) & $<0.001$ & $129(6.2)$ & $123(95.3)$ & 0.005 \\
\hline Three & $299(3.2)$ & $291(97.3)$ & & $50(2.4)$ & $49(98.0)$ & \\
\hline Four or more & $174(1.9)$ & $172(98.9)$ & & $19(0.9)$ & $19(100.0)$ & \\
\hline \multicolumn{7}{|c|}{ Adjustment/anxiety disorders } \\
\hline No diagnosis & $8840(94.3)$ & $8197(92.7)$ & 0.10 & $1993(96.0)$ & $1791(89.9)$ & 0.61 \\
\hline Diagnosis & $537(5.7)$ & $508(94.6)$ & & $83(4.0)$ & $76(91.6)$ & \\
\hline \multicolumn{7}{|l|}{ Mood disorders } \\
\hline No diagnosis & $7841(83.6)$ & $7245(92.4)$ & $<0.001$ & $1838(88.5)$ & $1650(89.8)$ & 0.50 \\
\hline Diagnosis & $1536(16.4)$ & $1460(95.1)$ & & $238(11.5)$ & $217(91.2)$ & \\
\hline \multicolumn{7}{|c|}{ Schizophrenia/other psychosis } \\
\hline No diagnosis & $9024(96.2)$ & $8378(92.8)$ & 0.88 & $1963(94.6)$ & $1770(90.2)$ & 0.14 \\
\hline Diagnosis & $353(3.8)$ & $327(92.6)$ & & $113(5.4)$ & $97(85.8)$ & \\
\hline \multicolumn{7}{|c|}{ Other mental health disorders } \\
\hline No diagnosis & $9101(97.1)$ & $8446(92.8)$ & 0.51 & $1999(96.3)$ & $1802(90.1)$ & 0.10 \\
\hline Diagnosis & $276(2.9)$ & $259(93.8)$ & & $77(3.7)$ & $65(84.4)$ & \\
\hline
\end{tabular}

For privacy reasons, cell sizes of less than 11 are not reported. 
used to estimate predictive margins and $95 \%$ confidence intervals (CIs).

\section{Results}

There were 2076 PLWHA with Medicaid coverage only and 9377 enrollees with Medicare, of whom 6650 were covered by both Medicare and Medicaid (duals). Table 1 (panel 1) presents data on the percent of Medicare recipients, overall and by subgroup, meeting guidelines for number and timing of E\&M visits. Seventy-one percent of Medicare recipients with HIV were dually enrolled in the Medicaid program. Medicare enrollees were predominately male (90\%), with a median age of 52 . Slightly more than $17 \%$ were African American, 19\% Hispanic, and 59\% white. For $13 \%$ of the sample, their most experienced provider had fewer than 5 publicly funded HIV cases, while $63 \%$ visited providers with a caseload of 50 or more publicly insured patients with HIV/ AIDS. Forty percent of the Medicare enrollees had 1 or more comorbidities, and $13.6 \%$ had 2 or more comorbidities.

The second panel of Table 1 presents comparable data for the Medicaid sample. Similar to the Medicare sample, males predominate (73\%), but median age is slightly younger (49). Thirty-three percent were African American, $18 \%$ Hispanic, and $39 \%$ white. For $13 \%$ of the sample, their most experienced provider had fewer than 5 HIV cases with Medicare or Medicaid insurance, while $66 \%$ visited providers with a caseload of 50 or more patients with HIV/AIDS. Thirty-six percent of the Medicaid enrollees had 1 or more comorbidities, and $9.5 \%$ had 2 or more comorbidities.

Table 1 shows that a large share of Medicare (93\%) and Medicaid (90\%) patients had guideline-compliant numbers of visits. The unadjusted percentages in the first row of Table 2 show that $89 \%$ of Medicare and $89 \%$ of Medicaid patients who had the recommended numbers of visits, received spaced CD4 testing at least twice within 2 years. Spaced VL assays were performed at least twice in 2 years for $85 \%$ of Medicare and $82 \%$ of Medicaid patients. However, smaller proportions of both populations received recommended noncore screenings and immunizations. Only 47\% of Medicare and $34 \%$ of Medicaid patients received an annual flu shot. TB testing more frequently appeared in the claims for Medicaid (35\%) than for Medicare enrollees (19\%). Seventytwo percent of Medicare enrollees and 64\% of Medicaid enrollees received a lipid panel and more than $94 \%$ in either group received a glucose test. Fewer than half of women in either program were screened for cervical cancer in 2010. Unadjusted rates of Medicare and Medicaid beneficiaries receiving guideline care within all subgroups can be found in Appendix Table 1.

Table 2 shows adjusted rates of guideline compliance based on logistic regressions that controlled for enrollee age, gender, race, provider caseload, and mental and physical comorbidities. Appendix Table 2 contains odds ratios from the logistic regressions; Appendix Table 3 contains adjusted rates of compliance by all beneficiary subgroups.

Controlling for patients' demographic and clinical characteristics, enrollees seeing only non-HIV specialists ( $<5$ HIV patients) were significantly less likely to meet recommended levels for the core indicators. Only 60\% (CI 57.1-62.3) of Medicare patients and 45\% (CI 38.3-50.4) of Medicaid patients seeing only non-HIV specialists met visit guidelines, compared to $98 \%$ (CI 97.5-98.2) of Medicare enrollees and 97\% (CI 96.2-98.0) of Medicaid enrollees who had providers who treated 50 or more patients ( $p<0.001$ in both groups). Among Medicare enrollees who visited only providers with small HIV caseloads, only 78\% (CI 74.6-80.5) received CD4 guideline care and 76\% (CI 73.1-79.1) received guideline care for VL testing, both rates were significantly lower than the rates observed among enrollees seeing providers who treated 50 or more patients (91\% [CI 89.9-91.4] and 87\% [CI 85.9-87.6], respectively; $p<0.001$ in both cases). Only $75 \%$ (CI 67.2-83.0) of Medicaid enrollees seeing only lowvolume providers received CD4 tests, significantly fewer CD4 tests than those with higher volume providers $(90 \%$; CI 88.4-91.6). The difference in VL testing was not statistically significant.

Medicare patients not seeing HIV specialists had significantly lower $(p<0.001)$ rates of immunization against influenza (36\%; CI 32.2-39.0) than those seeing the highest volume HIV providers (47\%; CI 45.5-48.0). There were no significant differences by caseload in rates of influenza vaccination for Medicaid enrollees. Enrollees who had providers with more HIV experience were more likely to receive TB testing. Nine percent (CI 6.6-10.6) of Medicare enrollees seeing only low-volume providers, versus $22 \%$ (CI 21.023.2) seeing high-volume providers, received a TB test within a year $(p<0.001)$. The contrast was sharper for Medicaid enrollees: $13 \%$ (CI 6.8-19.0) of those seeing providers with fewer than 5 HIV patients and 39\% (CI 36.141.3 ) of those seeing providers whose caseload was in the highest category received a TB test $(p<0.001)$. Lipid screening did not differ between patients with access to more HIV-experienced providers and those with less HIVexperienced providers, in either the Medicare or Medicaid samples. Rates of glucose testing in Medicaid patients were significantly lower $(p=0.01)$ among those seeing lowvolume providers (90\%; CI 84.9-95.7) versus high-volume providers (96\%; CI 94.6-96.8), but we found no significant differences among Medicare patients. Female Medicaid enrollees seeing only providers with fewer than five HIV patients were significantly less likely $(p=0.004)$ to be screened for cervical cancer (19\%; CI 5.4-33.2) than those with access to high-volume providers (48\%; CI 43.4-53.2). Among women in Medicare, those who visited large-volume HIV providers were more likely to be screened annually for cervical cancer (44\%; CI 39.9-48.1), but this number was not significantly greater than the rate among the lowest volume group (35\%; CI 25.2-43.9).

\section{Discussion}

The ultimate goal of HIV treatment is viral suppression, both to maintain the health of the patient and to reduce onward transmission to sexual partners. However, even sustained viral suppression does not entirely mitigate the longterm consequences of HIV-related inflammation in the form of early senescence and end-organ disease-including but not limited to cardiovascular disease, disordered glucose metabolism, neurocognitive impairment, renal dysfunction, and some nonopportunistic malignancies. ${ }^{4,30}$

The findings of this article confirm that patients whose providers included physicians with larger HIV caseloads are not only more likely to receive higher quality HIV primary care 


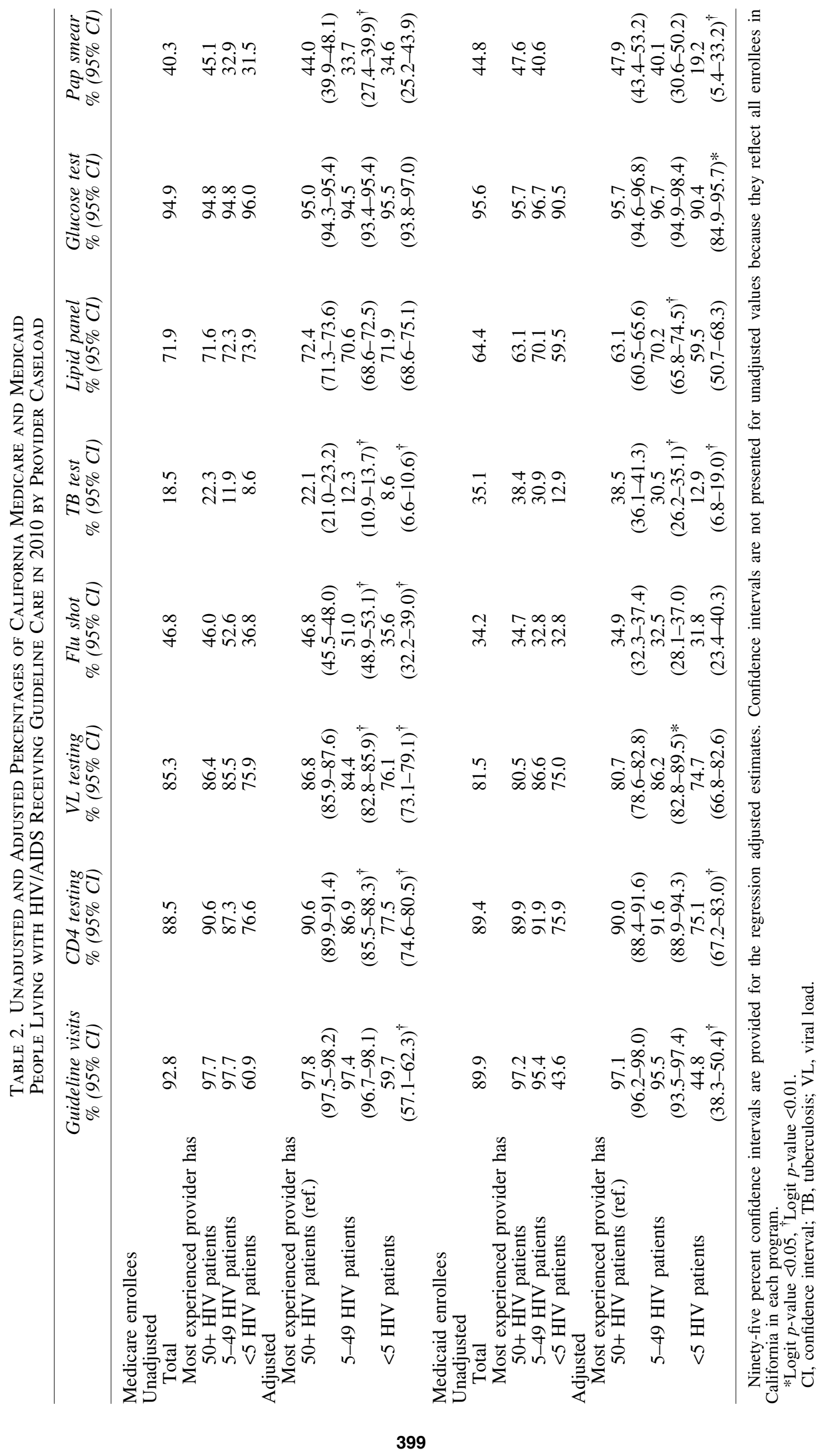


assessments (visits and CD4 and VL monitoring) but also ${ }^{12,14,15}$ were more likely to receive preventive care for conditions with greater prevalence among PLWHA (flu immunizations for Medicare patients, TB testing, cervical screening for women). Glucose testing was more frequently conducted for Medicaid patients. There were not significant differences in lipid assays.

The proportion of Medicare and Medicaid patients with HIV who achieved recommended numbers of visits is high, particularly among enrollees whose providers included HIV specialists with large HIV caseloads-98\% of Medicare enrollees and $97 \%$ of Medicaid enrollees. The value of frequent visits lies in providing the opportunity to monitor HIV treatment and to conduct preventive screening for other health issues. However, even among those patients with guideline-consistent visits, the rates of core HIV-specific care (CD4 and VL testing) are less than universal, in the range of 75-92\%, even among patients whose care team included HIV specialists. Rates of recommended CD4 and VL testing were lowest (75-78\%) among patients whose most experienced (as measured by patient volume) providers had small caseloads. Compared to the near-universal use of ART among Medicare $(87.3 \%)$ and Medicaid (96.2\%) enrollees, the low levels of CD4 and VL testing suggest insufficient levels of monitoring of disease status and need for/response to ART treatment. ${ }^{18}$ Treatment guidelines note that even PLWHA who were not on ART should be routinely evaluated. ${ }^{6}$

Screening rates for HIV-related comorbidities were also less than optimal. Despite the high rates of ART use, lipid testing was not frequently conducted among Medicare enrollees, either those on ART $(71 \%)$ or those not on ART $(76 \%)$. Glucose testing was uniformly high and nearly universal (96\%) across all provider sizes. Influenza immunization rates and TB testing rates were low.

Many of the comorbidities that an aging HIV/AIDS population is now experiencing fall outside of the scope of traditional HIV care and treatment, and the expertise of subspecialty-trained HIV providers. Some studies suggest that HIV specialists are uncomfortable providing treatment for other chronic diseases. ${ }^{31-33}$ This likely explains the finding that providers with greater HIV caseloads $(>50)$ and infectious disease trained specialists are more likely than generalists to refer HIV patients who also have hypertension or diabetes to other specialists. ${ }^{34}$ As comorbidities increase in the aging HIV population, care may increasingly be managed by teams composed of different subspecialists. However, our findings show that it is important that these teams include experienced HIV providers, because patients with access to these high-volume HIV providers were not only more likely to receive guideline-consistent HIV care but also more likely to be screened for other conditions associated with HIV, such as TB. Further, the high levels of visit frequency to HIV specialists provide them a unique opportunity to identify comorbidities and to deliver preventive services to PLWHA.

The generalizability of our findings is limited by the exclusion of PLWHA in managed care or with private insurance only. However, Medicare and Medicaid account for over half of PLWHA who are receiving care, ${ }^{35,36}$ minimizing the impact of this limitation. Second, the strict criteria we applied to identify $\mathrm{PLWHA}^{27}$ may have yielded a sample receiving better-than-average care. Third, we could not assess the delivery of services that are recommended once in a lifetime, such as hepatitis B vaccination. Our analysis also has a number of strengths. It is based on larger numbers of HIVpositive patients in geographically dispersed treatment settings, rather than on a small number of patients in a limited number of clinics.

It has previously been unclear whether patients whose primary care is driven by HIV specialty providers receive the preventive services that they need. Our results suggest that HIV specialists have weathered the evolution, over the past 30 years of the HIV epidemic, of HIV care from a practice largely focused on opportunistic infection management and end-of-life care, to one of highly specialized and nuanced antiretroviral management, to the current era ever-more focused on preventive care and long-term toxicity management.

Despite the near-universal use of ART in this population of publicly insured PLWHA, our results demonstrate substantial gaps in routine monitoring to assess viral suppression, even among patients with the most experienced providers. Patients with access to experienced HIV providers were as likely or more likely as others to receive routine assessments of common comorbidities, but rates at which these screens occurred were suboptimal.

Using provider education, automated EMR-based reminders, or other mechanisms to assure that primary care for PLWHA includes assessments of both HIV-specific outcomes and screening for comorbidities could have the greatest payoff among the providers with low caseloads of HIV patients. However, even the largest HIV providers are missing opportunities to provide comprehensive monitoring and preventive care for their $\mathrm{HIV}$-infected patients. The large gaps that remain between actual and recommended levels of preventive services suggest that even the highest volume providers need to increase guideline fidelity of their assessments for latent TB, cervical cancer, and dyslipidemia, and administration of influenza vaccine.

\section{Acknowledgments}

This research was supported by grants from the California HIV/AIDS Research Program (CHRP) of the University of California (grant RP11-LA-020). This work was also supported by the Center for HIV Identification, Prevention, and Treatment (CHIPTS) NIMH grant MH58107; the UCLA Center for AIDS Research (CFAR) grant 5P30AI028697; and the NIH/National Center for Advancing Translational Science (NCATS) UCLA CTSI Grant Number UL1TR000124. Research was approved by UCLA IRB \#10-000823. The funders had no role in data collection, management, analysis, interpretation of the data, preparation, review, or approval of the article for publication.

\section{Authors' Contributions}

R.J.L., K.A.D., and A.A.L. conceptualized the study. K.A.D. and J.L.G. were responsible for the statistical analysis. All authors contributed to the interpretation of the data and to the drafting and revising of the article. All authors approved the final version of the article.

\section{Author Disclosure Statement}

No competing financial interests exist. 


\section{References}

1. Samji H, Cescon A, Hogg RS, et al. Closing the gap: Increases in life expectancy among treated HIV-positive individuals in the United States and Canada. PLoS One 2013;8:e81355.

2. Legarth RA. Ahlström MG, Kronborg G, et al. Long-term mortality in HIV-infected individuals 50 years and older: A nationwide, population-based cohort study. J Acquir Immune Defic Syndr 2016;71:213-218.

3. Aberg JA, Kaplan JE, Libman H, et al. Primary care guidelines for the management of persons infected with human immunodeficiency virus: 2009 update by the HIV medicine Association of the Infectious Diseases Society of America. Clin Infect Dis 2009;49:651-681.

4. Appay V, Kelleher AD. Immune activation and immune aging in HIV infection. Curr Opin HIV AIDS 2016;11:242-249.

5. HIV/AIDS Bureau, Health Resources and Services Administration. HAB HIV Performance Measures, July 2008. Available at: http://hab.hrsa.gov/deliverhivaidscare/habperformmeasures. html (Last accessed January 20, 2016).

6. AIDS Info. NIH. Guidelines for the Use of Antiretroviral Agents in HIV-1-Infected Adults and Adolescents, January 28, 2016. Available at: https://aidsinfo.nih.gov/guidelines/ $\mathrm{html} / 1 /$ adult-and-adolescent-treatment-guidelines (Last accessed February 16, 2016).

7. Workowski KA, Bolan GA. Sexually transmitted diseases treatment guidelines, 2015. MMWR Recomm Rep 2015; 64(RR-3):1-137.

8. Rahangdale L, Sarnquist C, Yavari A, Blumenthal P, Israelski $\mathrm{D}$. Frequency of cervical cancer and breast cancer screening in HIV-infected women in a county-based HIV clinic in the Western United States. J Womens Health (Larchmt) 2010;19:709-712.

9. Reinhold J-P, Moon M, Tenner CT, Poles MA, Bini EJ. Colorectal cancer screening in HIV-infected patients 50 years of age and older: Missed opportunities for prevention. Am J Gastroenterol 2005;100:1805-1812.

10. Leece P, Kendall C, Touchie C, Pottie K, Angel JB, Jaffey J. Cervical cancer screening among HIV-positive women. Can Fam Physician 2010;56:e425-e431.

11. Stein MD, Cunningham WE, Nakazono T, et al. Screening for cervical cancer in HIV-infected women receiving care in the United States. J Acquir Immune Defic Syndr 2001; 27:463-466.

12. Kitahata MM, Koepsell TD, Deyo RA, et al. Physicians' experience with the acquired immunodeficiency syndrome as a factor in patients' survival. N Engl J Med 1996;334:701-706.

13. O'Neill M, Karelas GD, Feller DJ, Knudsen-Strong E, Lajeunesse D, Tsui D, et al. The HIV workforce in New York State: Does patient volume correlate with quality? Clin Infect Dis 2015;61:1871-1877.

14. Handford CD, Rackal JM, Tynan AM, Rzeznikiewiz D, Glazier RH. The association of hospital, clinic and provider volume with HIV/AIDS care and mortality: Systematic review and meta-analysis. AIDS Care 2012;24:267-282.

15. Rackal JM, Tynan AM, Handford CD, et al. Provider training and experience for people living with HIV/AIDS. Cochrane Database Syst Rev 2011:CD003938; DOI: 10.1002/ 14651858.CD003938.pub2.

16. Stone VE, Mansourati FF, Poses RM, Mayer KH. Relation of physician specialty and HIV/AIDS experience to choice of guideline-recommended antiretroviral therapy. J Gen Intern Med 2001;16:360-368.
17. Landon BE, Wilson IB, McInnes K, et al. Physician specialization and the quality of care for human immunodeficiency virus infection. Arch Intern Med 2005;165:1133-1139.

18. Landovitz RJ, Desmond KA, Leibowitz AA. Antiretroviral therapy: Racial disparities among publicly insured Californians with HIV. J Health Care Poor Underserved. Feb. 2017;28(1), in press. [Epub ahead of print].

19. Wilson IB, Landon BE, Hirschhorn LR, et al. Quality of HIV care provided by nurse practitioners, physician assistants, and physicians. Ann Intern Med 2005;143:729-736.

20. Landon BE, Wilson IB, Wenger NS, et al. Specialty training and specialization mong physicians who treat HIV/ AIDS in the United States. J Gen Intern Med 2002;17: 12-22.

21. Emdin CA, Chong NJ, Millson PE. Non-physician clinician provided HIV treatment results in equivalent outcomes as physician-provided care: A meta-analysis. J Int AIDS Soc 2013;16:18445.

22. Kendall CE, Taljaar M, Younger J, et al. A populationbased study comparing patterns of care delivery on the quality of care for persons living with HIV in Ontario. BMJ Open 2015;5:e007428.

23. Mgbere O, Rodriguez-Barrada MC, Bell TK, et al. Frequency and determinants of preventive care counseling by HIV medical care providers during encounters with newly diagnosed and established HIV-infected patients. J Int Assoc Provid AIDS Care 2016;15:215-227.

24. Sheth AN, Moore RD, Gebo KA. Provision of general and HIV-specific health maintenance in middle aged and older patients in an urban HIV clinic. AIDS Patient Care STDs 2006;20:318-325.

25. Curtis JR, Paaue FDS, Wenrich MD, Carline JD, Ramsey PG. Physicians' ability to provide initial primary care to an HIV-infected patient. Arch Intern Med 1995;155:16131618.

26. Koethe JR, Moore RD, Wagner KR. Physician specialization and women's primary care services in an urban HIV clinic. AIDS Patient Care STDs 2008;22:373-380.

27. Leibowitz AA, Desmond K. Identifying a sample of HIVpositive beneficiaries from Medicaid claims data and estimating their treatment costs. Am J Public Health 2015; 105:567-574.

28. U.S. Agency for Healthcare Research and Quality, Healthcare Cost and Utilization Project. Clinical Classifications Software (CCS) for ICD-9-CM. Available at: www. hcup-us.ahrq.gov/toolssoftware/ccs/ccs.jsp (Last accessed December 20, 2015).

29. Charlson ME, Pompei P, Ales KL, MacKenzie CR. A new method of classifying prognostic comorbidity in longitudinal studies: Development and validation. J Chronic Dis 1987;40:373-383.

30. Deeks SG, Tracy R, Douek DC. Systemic effects of inflammation on health during chronic HIV infection. Immunity 2013;39:633-645.

31. Bess KD, Adams J, Watt MH, et al. Providers' attitudes towards treating depression and self-reported depression treatment practices in HIV outpatient care. AIDS Patient Care STDs 2013;27:171-180.

32. Fultz S1, Goulet JL, Weissman S, et al. Differences between infectious diseases-certified physicians and general medicinecertified physicians in the level of comfort with providing primary care to patients. Clin Infect Dis 2005;41: 738-743. 
33. Cheng QJ, Engelage EM, Grogan TR, Currier JS, Hoffman RM. Who provides primary care? An assessment of HIV patient and provider practices and preferences. J AIDS Clin Res 2014;5:pii. 366.

34. Duffus WA, Barragan M, Metsch L, et al. Effect of physician specialty on counseling practices and medical referral patterns among physicians caring for disadvantaged human immunodeficiency virus-infected populations. Clin Infect Dis 2003;36:1577-1584.

35. Kaiser Family Foundation. U.S. federal funding for HIV/ AIDS: The president's FY2016 budget request. HIV/AIDS policy fact sheet. 2012. Available at: http://kff.org/globalhealth-policy/fact-sheet/u-s-federal-funding-for-hivaids-thepresidents-fy-2016-budget-request (Last accessed March 28, 2016).
36. Yehia BR, Fleishman JA, Agwu AL, et al. HIV Research Network. Health insurance coverage for persons in HIV care, 2006-2012. J Acquir Immune Defic Syndr 2014;67: $102-106$

Address correspondence to: Arleen A. Leibowitz, PhD Department of Public Policy UCLA Luskin School of Public Affairs Box 951656 3250 Public Affairs Building Los Angeles, CA 90095-1656

E-mail: leibowia@luskin.ucla.edu 


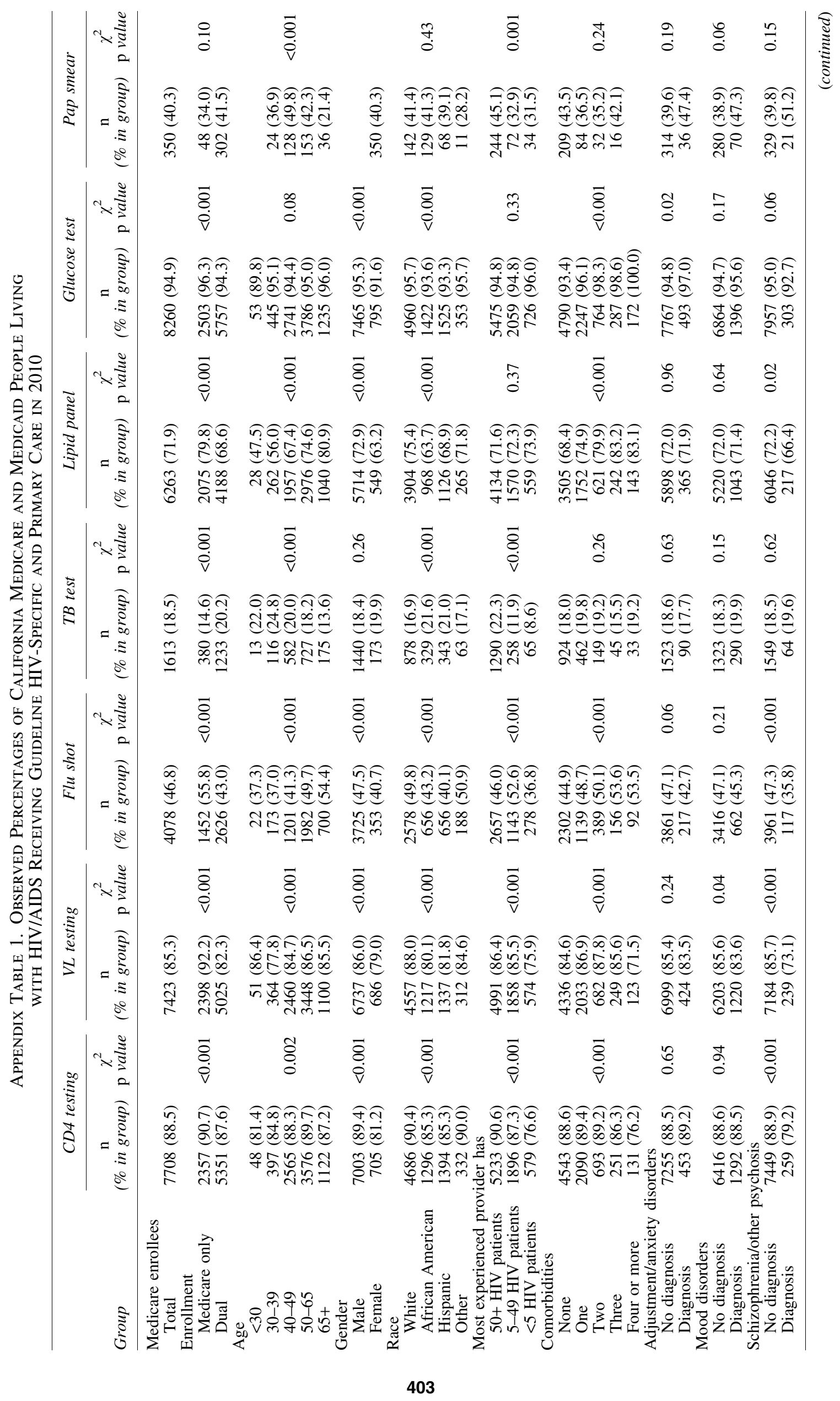




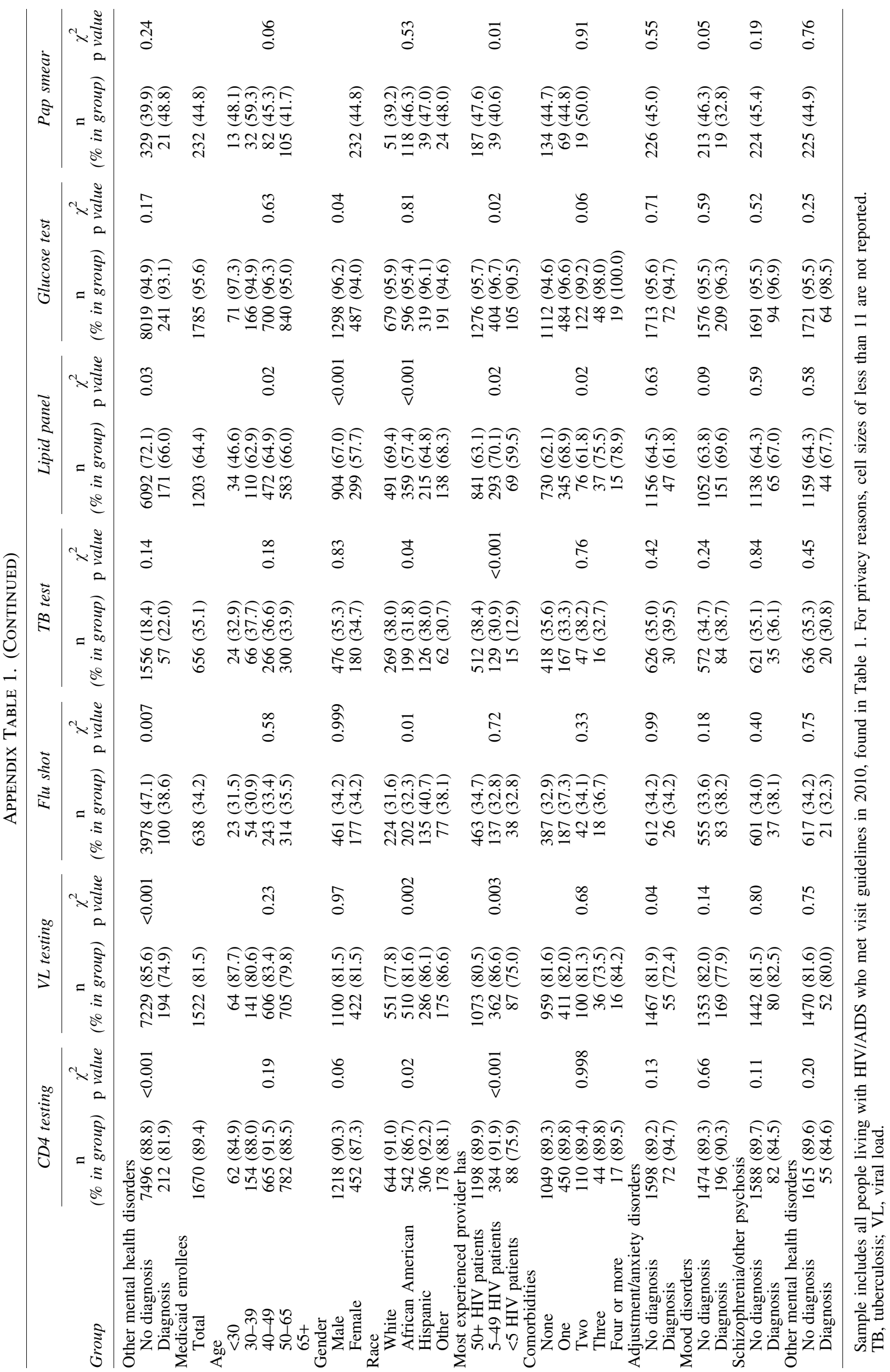




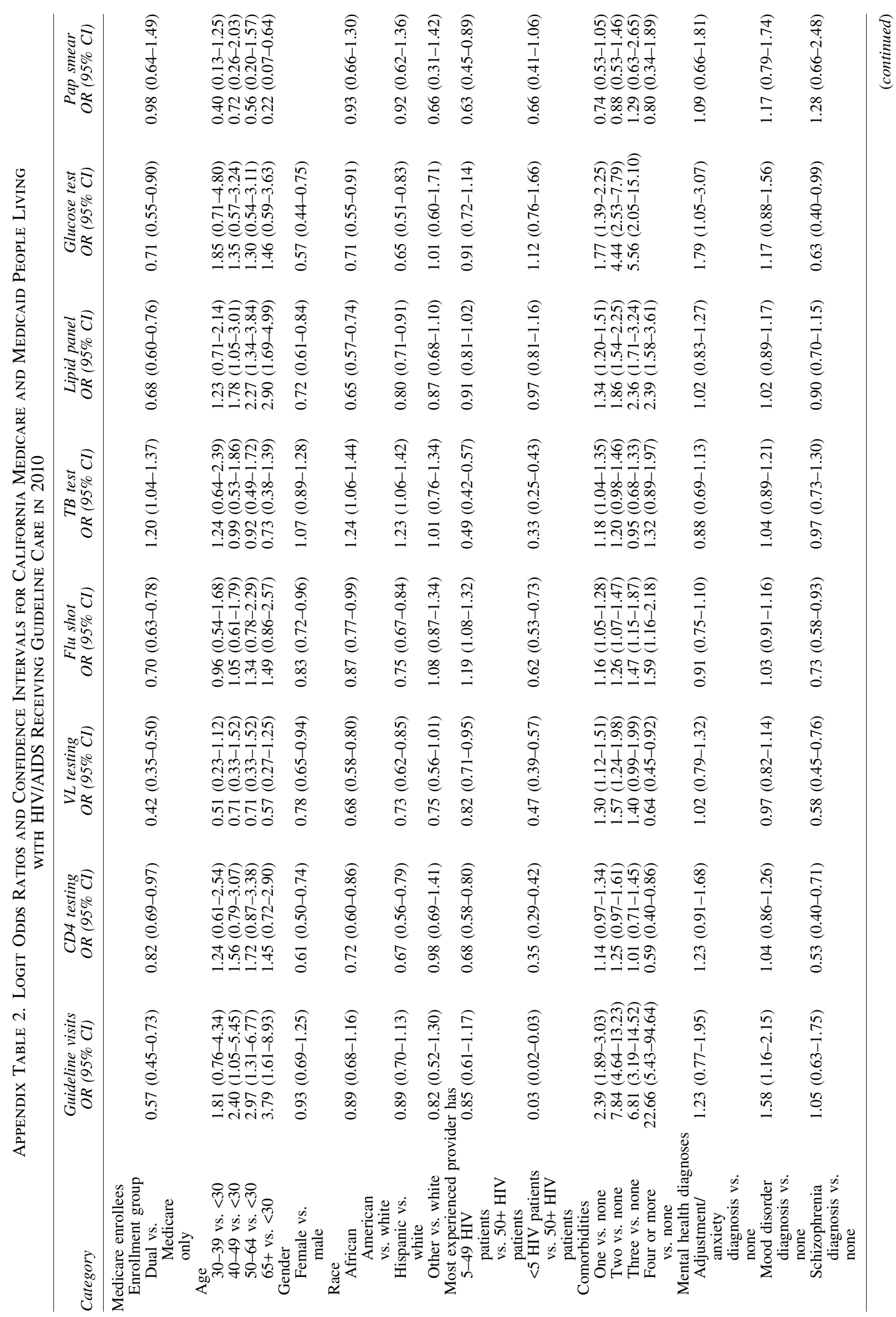




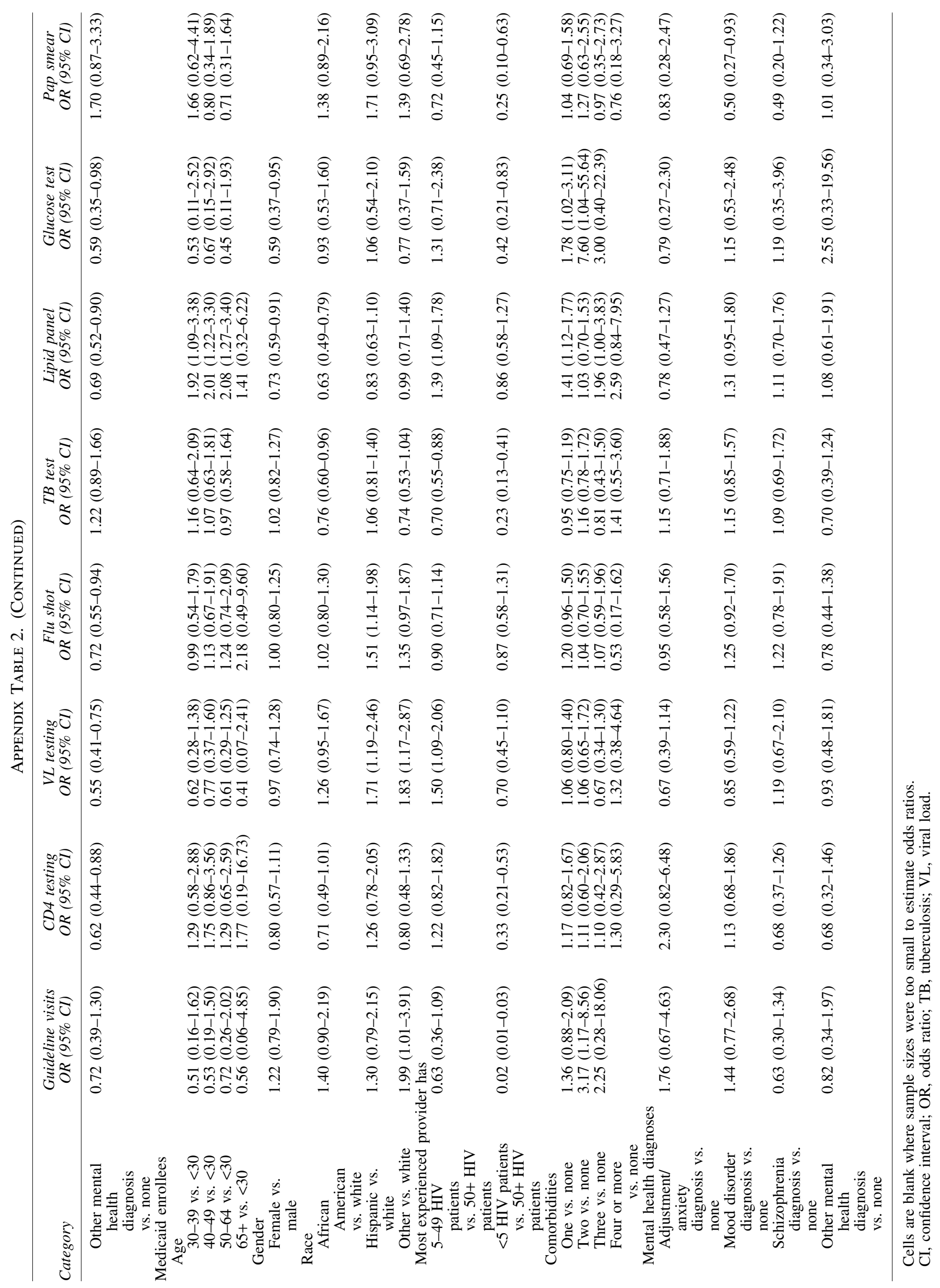




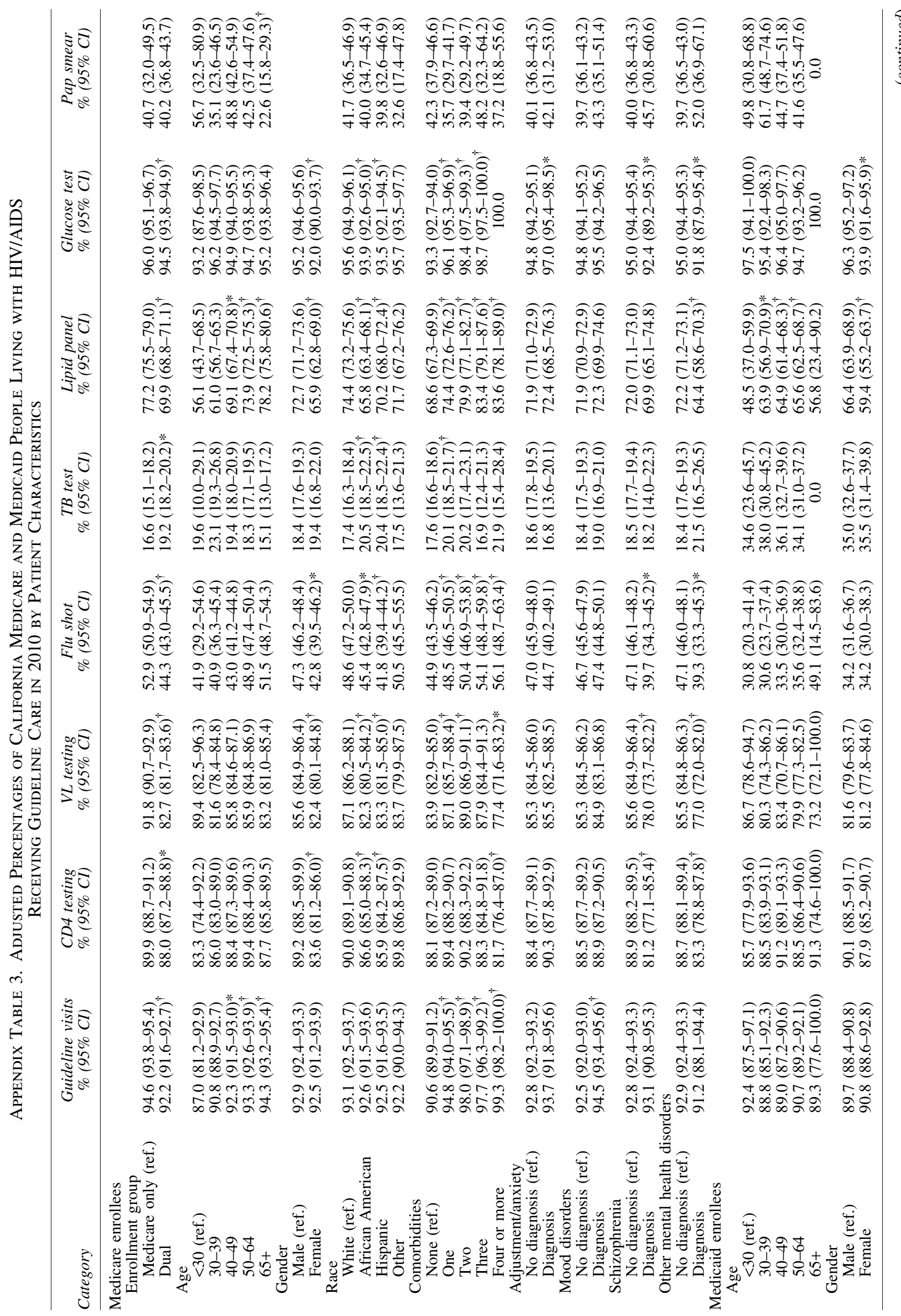




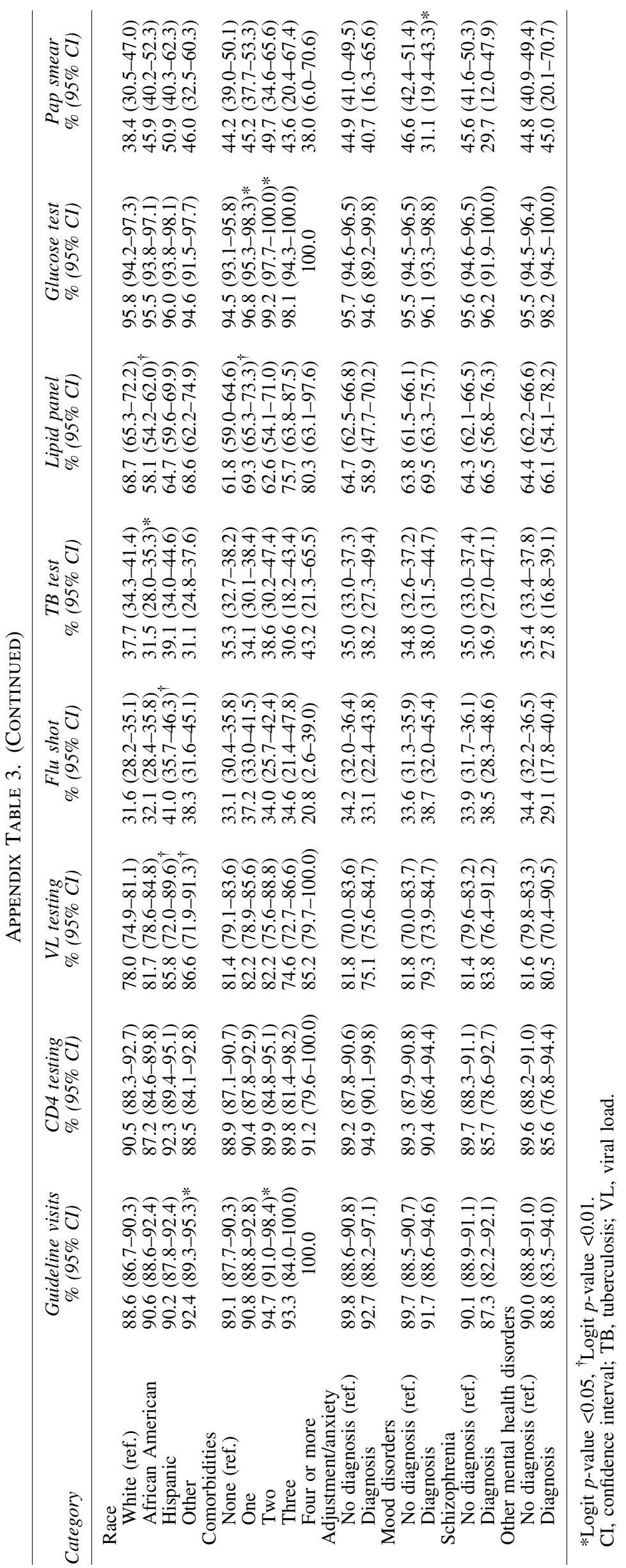

